O-0

\title{
Round-Robin Test of Medium-Energy Ion Scattering for Quantitative Depth Profiling of Ultrathin $\mathrm{HfO}_{2} / \mathrm{SiO}_{2} / \mathrm{Si}$ Films
}

\author{
Won Ja Min ${ }^{1}$, Gabriel M. Marmitt ${ }^{2}$, RRT Participants, Pedro L. Grande², and DaeWon Moon ${ }^{3}$ \\ ${ }^{1}$ MEIS dev. Team. K-MAC, Techno 8-ro 33, Yuseong, Daejeon 34028, Korea \\ ${ }^{2}$ Ion Implantation Laboratory, Institute of Physics, Federal University of Rio Grande do Sul, Av. Bento Gonçalves 9500, CP 15051, \\ CEP 91501-970, Porto Alegre, RS, Brazil \\ ${ }^{3}$ Department of New Biology, DGIST, Techno-JungAngDaeRo 333, Dalseong, Daegu 42988, Korea \\ *corresponding author's e-mail: dwmoon@dgist.ac.kr \& grande@if.ufrgs.br
}

(Received: May 20, 2019; Accepted: July 2, 2019)

\begin{abstract}
Medium energy ion scattering (MEIS) has been used for quantitative depth profiling of ultrathin films with single atomic layer resolution. To assure the consistency of the MEIS analysis, an international round-robin test with nominally $1,3,5$, and $7 \mathrm{~nm}$ thick $\mathrm{HfO}_{2}$ films was conducted among 12 institutions. The standard deviations were $5.3 \%$ for the composition, $15.3 \%$ for the thickness, and $13.3 \%$ for the Hf content by using stopping and range of ions in matter (SRIM) 95 , and they were improved to $7.3 \%, 4.5 \%$, and $7.0 \%$ by using refitted electronic stopping powers based on the experimental data. This study suggests that correct electronic stopping powers are critical for quantitative MEIS analysis.
\end{abstract}

\section{Medium Energy Ion Scattering}

Medium energy ion scattering (MEIS) is a surface analysis technique that uses medium-energy (100-500 $\mathrm{keV}$ ) ions. When incident ions hit the sample, the ions are scattered by the target nuclei of the sample. The scattered energy of the ions is reduced by the kinematic factor, which is determined by the mass ratio of the incident ion and the target nucleus, and the scattering angle. Additional energy loss occurs during ion beam path by the stopping power before and after the collision. The scattering yield of each element is determined as a function of concentration and the scattering cross section. As a result, the elemental concentration depth distributions in thin films is determined by measuring the scattering yield as a function of scattered ion energies.[1]-[2]

\section{Round-Robin Test (RRT)}

Nominally $1,3,5$, and $7 \mathrm{~nm}$ thick $\mathrm{HfO}_{2}$ films were deposited by atomic layer deposition on a $12 \mathrm{~nm}$ thermal $\mathrm{SiO}_{2}$ layer. The thermal $\mathrm{SiO}_{2}$ layer is used as an internal reference in analysis. The measurements were performed at each participating laboratory under their own conditions and the collected data were analyzed at K-MAC by using powermeis program[3]. Participants are presented at Table 1.

Table 1. List of participants in the round-robin test

\begin{tabular}{ll}
\hline Participants & Organization \\
\hline L. Goncharova & Western University, Canada \\
\hline K. Kimura & Kyoto University, Japan \\
\hline T. Gustafsson & Rutgers University, USA \\
\hline M. Copel & IBM Watson, USA \\
\hline J. Ko & SK hynix, Korea \\
\hline H.-I. Lee & SAIT, Korea \\
\hline S. Lee & Samsung Electronics, Korea \\
\hline K. Chae & KIST, Korea \\
\hline J. van den Berg & University of Huddersfield, UK \\
\hline K. Jung & DGIST, Korea \\
\hline K. Park & KMAC, Korea \\
\hline G. Marmitt & UFRGS, Brazil
\end{tabular}

For the data analysis, the Moliere potential[4] for scattering cross section, and the Chu [5] for straggling were used. For the electronic stopping power, the MEIS results obtained using the SRIM 95[6] values and the ones corrected with the IAEA data[7] were compared. For analyzing the MEIS data from the magnetic sector and electrostatic analyzers, the neutralization corrections of Marion and Young [8] for $100 \mathrm{keV} \mathrm{H}^{+}$and $\mathrm{He}^{+}$ions and of Armstrong[9] for $400-500 \mathrm{keV} \mathrm{He}{ }^{+}$ 

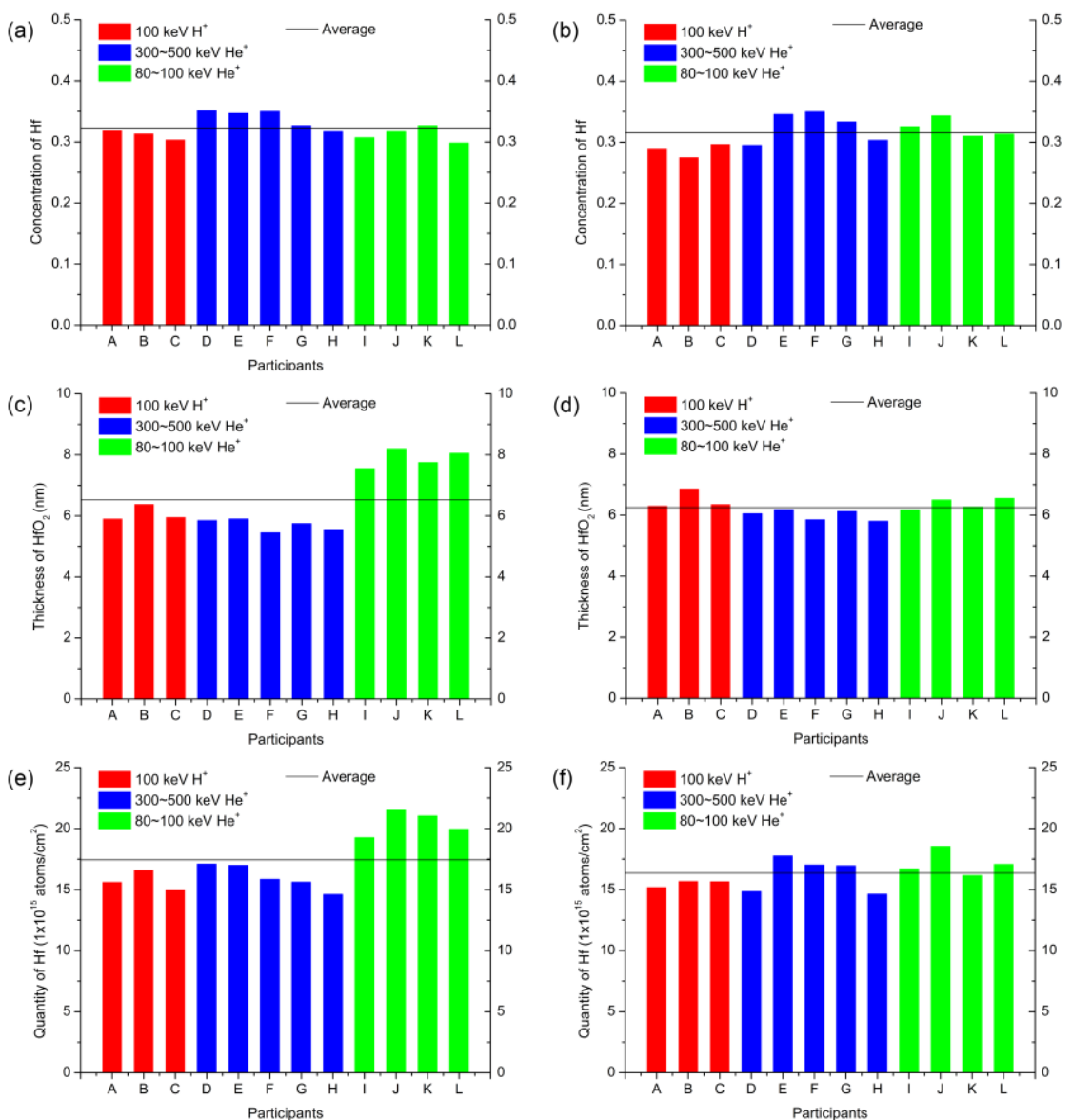

Fig. 1. Comparison of results obtained for the $7 \mathrm{~nm} \mathrm{HfO}_{2}$ sample: Hf concentration with (a) SRIM 95 and (b) new fitted electronic stopping power; $\mathrm{HfO}_{2}$ layer thickness with (c) SRIM 95 and (d) new fitted electronic stopping power; and Hf surface areal density with (e) SRIM 95 and (f) new fitted electronic stopping power.

ions were used. As a result, the concentration of $\mathrm{Hf}$, thickness of the $\mathrm{HfO}_{2}$ layer, the quantity of $\mathrm{Hf}$ were obtained, and results of the $7 \mathrm{~nm} \mathrm{HfO}_{2}$ sample are presented at Fig. 1 among all data. The average values were $32.3 \%$ for $\mathrm{Hf}$ concentration, $6.52 \mathrm{~nm}$ for $\mathrm{HfO}_{2}$ layer thickness, and $1.74 \times 10^{16}$ atoms $/ \mathrm{cm}^{2}$ for $\mathrm{Hf}$ areal density by using SRIM95 stopping power. The standard deviations were $5.3 \%$ for the composition, $15.3 \%$ for the thickness, and $13.3 \%$ for the Hf areal density. When they are analyzed by using refitted electronic stopping powers based on the experimental data[7], the average values were $31.5 \%$ in $\mathrm{Hf}$ concentration, $6.25 \mathrm{~nm}$ in $\mathrm{HfO}_{2}$ layer thickness, and $1.63 \times 10^{16}$ atoms $/ \mathrm{cm}^{2}$ in $\mathrm{Hf}$ areal density, and the standard deviations were improved to $7.3 \%, 4.5 \%$, and $7.0 \%$ respectively.

\section{Conclusion}

The RRT was conducted to investigate the consistency in the concentration of $\mathrm{Hf}$, the $\mathrm{HfO}_{2}$ layer thickness, and the surface areal density of $\mathrm{Hf}$ determined by MEIS analysis for nominally 1, 3, 5, and
$7 \mathrm{~nm}$ thick $\mathrm{HfO}_{2}$ films. As a result, the corrected electronic stopping power values provided more consistent results with relative standard deviations of $2-8.2 \%$, compared to the SRIM 95 values, with relative standard deviations of 5.3-15\%, for the Hf concentration, the $\mathrm{HfO}_{2}$ layer thickness, and surface areal density.

\section{References}

[ 1] E. P. Gusev et al., App. Phys. Lett. 76, 176 (2000).

[2] M. M. Frank et al., App. Phys. Lett. 86, 152904 (2005).

[3] M. Sortica et al., J. Appl. Phys., 106, 114320 (2009).

[4] G. Moliere, Z. Naturforsch., 2a, 133 (1947).

[5] W. K. Chu, Phys. Rev. A, 13, 2057 (1976).

[6] http://www.srim.org/

[7] https://www-nds.iaea.org/stopping/

[8] J. B. Young et al., Nuclear Reaction AnalysisGraphs and Tables, North Holland, Amsterdam, (1968)

[9] J. C. Armstrong et al. Proc. Phys. Soc., 86, 1283 (1965). 